\title{
Chiral Symmetry in Nuclei
}

\author{
Kuniharu Kubodera ${ }^{\text {a* }}$ \\ ${ }^{a}$ Department of Physics and Astronomy, University of South Carolina, \\ Columbia, South Carolina 29208, USA
}

Effective field theory is considered to provide a highly useful framework for connecting nuclear physics with the symmetries and dynamics of the underlying theory of strong interactions, QCD. Of many issues that are of great current interest in this domain, I concentrate here on two: (1) A new class of ab initio calculations of observables in twonucleon systems; (2) Attempts to extend chiral perturbation calculations to higher-order terms.

\section{Introduction}

One of the major challenges in nuclear physics today is to establish a connection between nuclear dynamics and the fundamental QCD. Effective field theory (EFT) provides a natural and useful framework for this purpose. The basic idea of EFT is simple [1.2]. Suppose we are interested in phenomena characterized by a typical energy-momentum scale $Q$. We expect that the degrees of freedom whose energy scales are significantly larger than $Q$ need not feature explicitly in our Lagrangian. So, introducing a cut-off scale $\Lambda$ that is reasonably large as compared with $Q$, we separate our fields (generically denoted by $\Phi$ ) into a high-energy part $\Phi_{\mathrm{H}}$ and a low-energy part $\Phi_{\mathrm{L}}$. Integrating out $\Phi_{\mathrm{H}}$, we arrive at an effective Lagrangian that only involves $\Phi_{\mathrm{L}}$. The original Lagrangian $\mathcal{L}$ and the effective Lagrangian $\mathcal{L}_{\text {eff }}$ are related as

$$
\int[d \Phi] \mathrm{e}^{\mathrm{i} \int d^{4} x \mathcal{L}(\Phi)}=\int\left[d \Phi_{\mathrm{L}}\right] \mathrm{e}^{\mathrm{i} \int d^{4} x \mathcal{L}_{\text {eff }}\left(\Phi_{\mathrm{L}}\right)}
$$

By construction, $\mathcal{L}_{\text {eff }}$ inherits all the symmetries (and the patterns of symmetry breaking, if any) of the original $\mathcal{L}$. Then $\mathcal{L}_{\text {eff }}$ is given as the sum of all possible monomials of $\Phi_{\mathrm{L}}$ and their derivatives that are consistent with the symmetry requirements. Since a term involving $n$ derivatives scales like $(Q / \Lambda)^{n}$, we have perturbative expansion with respect to $\partial_{\mu} / \Lambda$. In a nuclear-physics application of EFT, the original Lagrangian $\mathcal{L}$ is the QCD Lagrangian, but, if we are concerned with the energy-momentum regime $Q \ll \Lambda_{\mathrm{QCD}} \sim 1$ $\mathrm{GeV}$, the relevant effective degrees of freedoms are not quarks and gluons but hadrons. Furthermore, for $Q \leq m_{\pi}$, it is reasonable to retain only the pions and nucleons as effective degrees of freedom. Meanwhile, chiral symmetry of QCD must be respected in our effective world. The resulting effective theory is called chiral perturbation theory

*e-mail: kubodera@sc.edu. Supported in part by the National Science Foundation, USA, Grant No.PHYS-9602000 
$(\chi \mathrm{PT})$ [2,3]. In fact, the inclusion of the nucleon in $\chi \mathrm{PT}$ poses a problem because its mass is comparable to $\Lambda_{\mathrm{QCD}}$. The heavy-baryon chiral perturbation formalism ( $\left.\mathrm{HB} \chi \mathrm{PT}\right)$ allows us to circumvent this difficulty [4,5]. $\mathcal{L}_{\mathrm{ch}}^{\mathrm{HB}}$ has, as effective degrees of freedom only the pions and the large components of nucleons, and it involves expansion in $\partial_{\mu} / \Lambda_{\mathrm{QCD}}$, $m_{\pi} / \Lambda_{\mathrm{QCD}}$ and $\partial_{\mu} / m_{\mathrm{N}}$. Since $m_{\mathrm{N}} \approx \Lambda_{\mathrm{QCD}}$, we usually lump together chiral and heavybaryon expansions. In this combined expansion scheme, the effective chiral Lagrangian can be organized in terms of the chiral order index $\bar{\nu}$ defined by $\bar{\nu}=d+(n / 2)-2$, where $n$ is the number of fermion lines that participate in a vertex, and $d$ is the number of derivatives (with $m_{\pi}$ counted as one derivative). The leading order terms are given as [5]

$$
\begin{aligned}
\mathcal{L}^{(0)}= & \frac{f_{\pi}^{2}}{4} \operatorname{Tr}\left[\partial_{\mu} U^{\dagger} \partial^{\mu} U+m_{\pi}^{2}\left(U^{\dagger}+U-2\right)\right] \\
& +\bar{B}\left(i v \cdot D+g_{A} S \cdot u\right) B-\frac{1}{2} \sum_{A} C_{A}\left(\bar{B} \Gamma_{A} B\right)^{2} \\
\mathcal{L}^{(1)}= & -\frac{i g_{A}}{2 m_{\mathrm{N}}} \bar{B}\{S \cdot D, v \cdot u\} B+2 c_{1} m_{\pi}^{2} \bar{B} B \operatorname{Tr}\left(U+U^{\dagger}-2\right) \\
& +\left(c_{2}-\frac{g_{A}^{2}}{8 m_{\mathrm{N}}}\right) \bar{B}(v \cdot u)^{2} B+c_{3} \bar{B} u \cdot u B \\
& -\frac{c_{9}}{2 m_{\mathrm{N}}}(\bar{B} B)(\bar{B} i S \cdot u B)-\frac{c_{10}}{2 m_{\mathrm{N}}}\left(\bar{B} S^{\mu} B\right)\left(\bar{B} i u_{\mu} B\right) .
\end{aligned}
$$

Here $U(x)$ is an $\mathrm{SU}(2)$ matrix field related non-linearly to the pion field, $\xi \equiv \sqrt{U}, u_{\mu} \equiv$ $i\left(\xi^{\dagger} \partial_{\mu} \xi-\xi \partial_{\mu} \xi^{\dagger}\right), S_{\mu}=i \gamma_{5} \sigma_{\mu \nu} v^{\nu} / 2$, and $D_{\mu}$ is the covariant derivative acting on the nucleon. Here we have retained only those terms that are relevant to our subsequent discussion. The counting rule of Weinberg [3] is that a Feynman diagram consisting of $A$ nucleons, $N_{E}$ external fields, $L$ loops and $C$-separated pieces is order of $\mathcal{O}\left(Q^{\nu}\right)$ with $\nu=2 L+2(C-1)+2-\left(A+N_{E}\right)+\sum_{i} \bar{\nu}_{i}$.

In fact, straightforward chiral counting fails for a nucleus, because purely nucleonic intermediate states that occur in a nucleus can have very low excitation energies which invalidate the ordinary chiral counting rule [3]. To avoid this difficulty, we classify Feynman diagrams into two groups. Diagrams in which every intermediate state contains at least one meson in flight are called irreducible diagrams, and all others are called reducible diagrams. The chiral counting should be applied only to irreducible diagrams. The contribution of all the irreducible diagrams (up to a specified chiral order) is then used as an effective operator acting on the nucleonic Hilbert space. By summing up infinite series of irreducible diagrams (either solving the Schrödinger equation or the Lippman-Schwinger equation), we take account of reducible diagrams. This two-step procedure may be referred to as nuclear chiral perturbation theory [3, 6].

\section{Hybrid approach to nuclear $\chi \mathrm{PT}$}

In applying nuclear $\chi \mathrm{PT}$ to cases that involve external probes, a nuclear transition operator $\mathcal{T}$ is identified with a set of all the irreducible diagrams (up to a given chiral order $\nu$ ) with an external current inserted [8]. In a fully consistent $\chi \mathrm{PT}$ calculation, $\mathcal{T}$ is to be sandwiched between the initial and final nuclear states which are governed by the nucleon interactions corresponding to the $\nu$-th order irreducible diagrams. In 
practice, however, we often use initial and final nuclear wavefunctions obtained from the Schrödinger equation that involves phenomenological nucleon-nucleon interactions. This eclectic treatment may be called a hybrid approach to nuclear $\chi \mathrm{PT}$. The hybrid approach was used extensively to test the validity of the "chiral filter mechanism" [7],8]. This mechanism is the statement that soft-pion exchange (unless suppressed by symmetry or kinematics) should give a dominant exchange-current contribution. Rho [8] gave a clear interpretation of this dominance in the language of chiral counting. As argued in [7], the space component of the vector current $(\mathbf{V})$ and the time component of the axial current $\left(A_{0}\right)$, in general, are expected to exhibit this dominance. Regarding the $\mathbf{V}$, a detailed calculation based on the hybrid $\mathrm{HB} \chi \mathrm{PT}$ was carried out by Park, Min and Rho [9] for the isovector M1 transition amplitude in the $n$ (thermal) $+p \rightarrow d+\gamma$ reaction. The result of their next-to-next-to-leading order calculation indicates that the soft-pion exchange current indeed gives a dominant contribution and that, with the next order corrections added, agreement with the data is perfect. As for the $A_{0}$, the enhancement factors for first-forbidden $(\Delta J=0, \Delta \pi=$ yes $) \beta$-decay transitions were calculated in the hybrid $\mathrm{HB} \chi \mathrm{PT}$ [10. The results indicate that the bulk of the empirical enhancement factors 11] can be attributed to the soft-pion exchange current, and that the next-order corrections are a small fraction of it.

Furthermore, Park, Min, Rho and myself (PKMR) 12 have recently computed in $\mathrm{HB} \chi \mathrm{PT}$ the cross sections for the solar proton burning process $p+p \rightarrow d+e^{+}+\nu_{e}$. The $p p$ fusion rate obtained in PKMR's calculation essentially agrees with the rate used in the standard solar model [13]. This result is important in the light of Ivanov et al.'s recent claim [14 that their field theoretic approach gives a $p p$ fusion rate significantly different than that obtained from the ordinary nuclear physics approach based on Schrödinger equations. PKMR's result based on $\chi \mathrm{PT}$ does not support the claim in Ref.[14].

\section{First attempt at $a b$ initio calculation}

Despite the impressive success of the hybrid approach, it is important to formally justify or go beyond this approximation. Very recently we (PKMR) have carried out a calculation in which the transition operators and the nuclear interactions are treated on the same footing [15,16]. This formally consistent treatment may be called an ab initio calculation. Let me summarize here some salient features of our work described in [16].

We have performed next-to-leading-order (NLO) ab initio calculations for the twonucleon systems both with and without the pion field. The purpose of considering these two cases is to examine whether the "pionless" and "pionful" effective theories, which have different cut-off scales, exhibit behaviors that are generally expected for EFT.

Since, to NLO, pion loops do not enter, we can simply work with a potential. The bare potential $\mathcal{V}$ has the form

$$
\mathcal{V}(\mathbf{q})=-\tau_{1} \cdot \tau_{2} \frac{g_{A}^{2}}{4 f_{\pi}^{2}} \frac{\sigma_{1} \cdot \mathbf{q} \sigma_{2} \cdot \mathbf{q}}{\mathbf{q}^{2}+m_{\pi}^{2}}+\frac{4 \pi}{m_{\mathrm{N}}}\left[C_{0}+\left(C_{2} \delta^{i j}+D_{2} \sigma^{i j}\right) q^{i} q^{j}\right] \mathbf{q}^{2}
$$

with $\sigma^{i j}=3 / \sqrt{8}\left[\left(\sigma_{1}^{i} \sigma_{2}^{j}+\sigma_{1}^{j} \sigma_{2}^{i}\right) / 2-\left(\delta^{i j} / 3\right) \sigma_{1} \cdot \sigma_{2}\right]$, where $\mathbf{q}$ is the momentum transfer. The parameters $C$ 's and $D_{2}$ are defined for each isospin channel. The first (nonlocal) term is the pion exchange involving the Goldstone boson and hence completely known 
from $\chi \mathrm{PT}$. The (local) terms in the square brackets represents the effects of the degrees of freedom that have been integrated out. Use of the potential Eq.(4) in the LippmanSchwinger equation generates an infinite series of divergent terms. How to regularize this divergence is one of the hottest issues in nuclear $\chi \mathrm{PT}$ (see blow). We use a momentum cutoff scheme [17] and introduce a Gaussian cutoff: $V(\mathbf{r}) \equiv \int\left[d^{3} \mathbf{q} /(2 \pi)^{3}\right] \mathrm{e}^{i \mathbf{q} \cdot \mathbf{r}} S_{\Lambda}\left(\mathbf{q}^{2}\right) \mathcal{V}(\mathbf{q})$, with $S_{\Lambda}\left(\mathbf{q}^{2}\right)=\exp \left(-\mathbf{q}^{2} / 2 \Lambda^{2}\right)$. For a given cutoff we can determine the constants $C$ 's and $D$ 's in (4) by relating them (after renormalization) to the scattering length and the effective range for the scattering channels or to a selected set of the deuteron observables. Then we are in a position to predict the N-N scattering phase shifts and the low-energy properties of the deuteron (other than those used as input). Moreover, we can make parameter-free estimation of electroweak transition amplitudes. (In our NLO calculation this is limited to the 1-body (impulse-approximation) contributions.) For this we only need the one-nucleon electroweak current derived to leading order. We have evaluated one-body contributions to the charge radius $r_{d}$, the quadrupole moment $Q_{d}$, and the magnetic moment $\mu_{d}$ of the deuteron. Furthermore, we have computed the one-body M1 matrix element $\mathcal{M}_{\mathrm{M} 1}$ for the $n p$ capture process and the Gamow-Teller matrix element $\mathcal{M}_{\mathrm{GT}}$ for the $p p$ fusion process. The upshot of PKMR's ab initio calculations is as follows. (1) All the calculated quantities are in good agreement with the empirical information in both pionless and pionful cases. (2) The results are stable against the variation of the cut-off parameter $\Lambda$, so long as it lies within a reasonable range; this reasonable range is found to be $\Lambda=100-300 \mathrm{MeV}(\Lambda=200-500 \mathrm{MeV})$ in the absence (presence) of the pion, values that are consistent with the general EFT consideration. (3)The presence of the pion brings a noticeable improvement in the accuracy of the prediction, markedly reducing the cutoff dependency.

The above-mentioned "good agreement" requires a little more explanation. First, for the quantities that do not involve external probes, our calculated values can be compared directly with the experimental values, or with the values obtained from e.g. the Argonne $v 18$ potential (which was constructed to fit the data). The experimental phase shifts are reproduced very well up to $p \approx 70 \mathrm{MeV}$ without the pion and up to $p \approx 140 \mathrm{MeV}$ with the pion. The deuteron D-state probability corresponding to the $v 18$ potential is also reproduced satisfactorily. People may ask, however, what is the difference between the familiar effective-range formula and our prediction. It is true that, in the pionless case, the two low-energy constants, $C_{0}$ and $C_{2}$ in Eq.(任), practically replace the roles of the effective-range expansion parameters, $a$ and $r_{e}$. With introduction of the pion, however, there is no such trivial correspondence, and $\chi \mathrm{PT}$ expansion contains more physics in it. Furthermore, $\chi \mathrm{PT}$ enables us to apply a unified expansion scheme not only to N-N scattering but also to nuclear transition processes.

The quantities that involve electroweak probes may be classified into two kinds according to whether two-body contributions are expected to be very small or rather significant. To the first kind belong $r_{d}, Q_{d}$ and $\mu_{d}$, while $\mathcal{M}_{\mathrm{M} 1}$ and $\mathcal{M}_{\mathrm{GT}}$ are of the second kind. As for the first group, PKMR's results with the one-body current alone agree well with the experimental values. As for $\mathcal{M}_{\mathrm{M} 1}$ and $\mathcal{M}_{\mathrm{GT}}$, PKMR's results are found to be in good agreement with the one-body contributions calculated with the use of $v 18$ potential. This aspect is of course welcome but precise comparison with the experimental $\mathcal{M}_{\mathrm{M} 1}$ can be done only after inclusion of exchange-current contributions. (For $\mathcal{M}_{\mathrm{GT}}$ there is 
no experimental data.) Since a completely self-consistent evaluation of the exchangecurrent, which requires an NNLO calculation, is yet to be done, we may proceed as follows. We introduce the ratio $\mathcal{R}$ of the 2-body contribution to the 1-body contribution by $\mathcal{M}_{\mathrm{M} 1}=\mathcal{M}_{\mathrm{M} 1}^{1 \text {-body }}+\mathcal{M}_{\mathrm{M} 1}^{\text {-body }} \equiv \mathcal{M}_{\mathrm{M} 1}^{1 \text {-body }}(1+\mathcal{R})$. Park, Min and Rho [9] calculated $\mathcal{R}$ in hybrid $\mathrm{HB} \chi \mathrm{PT}$ with the use of the $v 18$ potential and the resulting $\mathcal{M}_{\mathrm{M} 1}$ showed excellent agreement with the experimental value. Since the ratio $\mathcal{R}$ is very likely to be much less sensitive to the wavefunctions than $\mathcal{M}_{\mathrm{M} 1}$ itself, it is reasonable to expect that $\mathcal{R}$ calculated in the hybrid approach is close to $\mathcal{R}$ that would result from an ab initio evaluation. This expectation gets additional support from the fact that the range of the soft-pion exchange current is about the same as the range probed in our EFT. In this sense the hybrid approach is justifiable from the EFT point of view.

In the above we introduced a momentum cut-off to regularize the divergence that appears in the Lippman-Schwinger equation with a potential of the type Eq.(4). Another regularization method, called the polynomial divergence subtraction (PDS), was proposed by Kaplan, Savage and Wise [18], and it has been used extensively to calculate various observables in the two-nucleon systems [19,20]. One of the advantages of PDS is that it preserves chiral invariance. By contrast, the cut-off regularization loses manifest chiral invariance. Although this feature is not consequential in the NLO calculation discussed above, it does become relevant in higher-order calculations. Meanwhile, the PDS counting scheme generates a counter term that is of the same order as the leading-order pionexchange current 20]. Since the strength of this counter term needs to be determined using data, there is significant loss of predictivity. This is to be contrasted with the cutoff scheme in which the leading-order pion-exchange contribution is a prediction of the theory, and in which the problem of unknown counter terms occurs only in the loop corrections to the one-pion exchange term [9,10]. For more discussion on the regularization schemes in the two-nucleon systems, see e.g. [21].

\section{4. $p+p \rightarrow p+p+\pi^{0}$ reaction near threshold}

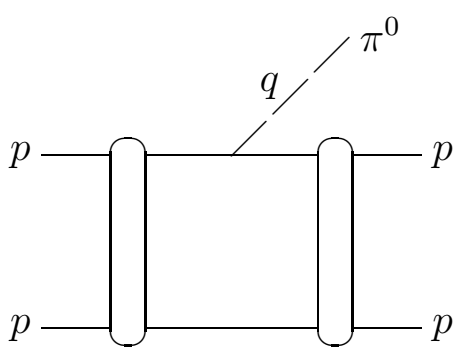

Fig. 1(a)

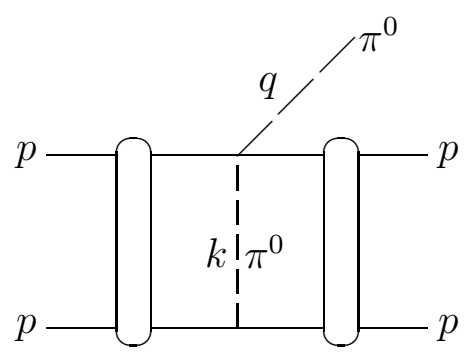

Fig. 1(b)

I next discuss the near-threshold $p p \rightarrow p p \pi^{0}$ reaction, which has recently been attracting a great deal of attention [22] - 29]. You may wonder what motivates us to study this very specific process. Besides the availability of high-precision data [22], a strong motivation comes from the exceptional sensitivity of this reaction to higher chiralorder terms. The corresponding charged-pion production reaction, e.g. $p p \rightarrow p n \pi^{+}$, is described reasonably well by the single nucleon process (Born term), Fig.1(a), and the "large" Weinberg-Tomozawa s-wave pion rescattering process, Fig.1(b). By contrast, the Weinberg-Tomozawa term does not contribute to the $p p \rightarrow p p \pi^{0}$ reaction, rendering it 
particularly sensitive to and hence a good testing ground for the less-well-understood "small" isoscalar s-wave pion rescattering terms.

The first $\chi \mathrm{PT}$ calculations for $p p \rightarrow p p \pi^{0}$ were carried out by Park et al. 25] and Cohen et al. [26]. In the hybrid $\mathrm{HB} \chi \mathrm{PT}$ they used, the transition amplitude is given by $T=\left\langle\Phi_{f}\left|\sum_{\nu} \mathcal{T}^{(\nu)}\right| \Phi_{i}\right\rangle$, where $\left|\Phi_{i}\right\rangle\left(\left|\Phi_{f}\right\rangle\right)$ is the initial (final) two-nucleon state distorted by the phenomenological N-N interaction. $\mathcal{T}^{(\nu)}$ stands for the transition operator of chiral order $\nu$. The lowest-order one-body impulse term [Fig.1(a)] gives $\mathcal{T}^{(\nu=-1)}$, while the lowest-order two-body rescattering term [Fig.1(b)] gives $\mathcal{T}^{(\nu=1)}$. The $\nu=1 \mathrm{HB} \chi \mathrm{PT}$ calculations in [25,26] lead to $p p \rightarrow p p \pi^{0}$ cross sections that are much smaller than the measured values. Meanwhile, Lee and Riska's work [23] based on the one-boson exchange $\mathrm{N}-\mathrm{N}$ potential suggests that shorter range isoscalar meson-exchange processes, like $\sigma$ and $\omega$ - exchanges between the two protons, might be very important for the $p p \rightarrow p p \pi^{0}$ reaction. To study the behavior of higher chiral-oder terms and to examine a possible connection between these higher order terms and the heavy-meson exchange contributions, it seems of great importance to perform a $\nu=2$ calculation. Such a calculation has recently been done by Dmitrasinovic, Myhrer, Sato and myself (DKMS) [29]. I describe here briefly the highlights of our results.

As an initial attempt we may concentrate on the effective transition operators $\mathcal{T}^{(\nu)}$ themselves instead of the full distorted-wave transition amplitude T. Furthermore, we limit ourselves to the threshold kinematics, which means that, in Fig. $1(\mathrm{~b}), q=\left(m_{\pi}, \overrightarrow{0}\right)$ and $k=\left(m_{\pi} / 2, \vec{k}\right)$ with $k^{2}=-m_{\pi} m_{N}$. Enumerating $\nu=2$ irreducible diagrams that give rise to transition operator $\mathcal{T}^{(\nu=2)}$ for $N N \rightarrow N N \pi$, we find 20 topologically distinct types of diagrams (nineteen of them are new). For a particular case of the $p p \rightarrow p p \pi^{0}$ reaction near threshold, the isospin selection rules and the s-wave character of the outgoing pion reduce this number from 20 to 7 (six of them are new). Some representative diagrams are depicted in Fig.2.

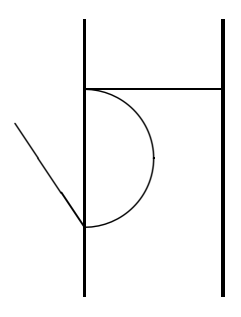

(a)

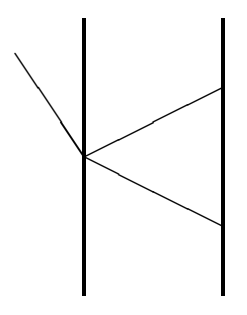

(b)

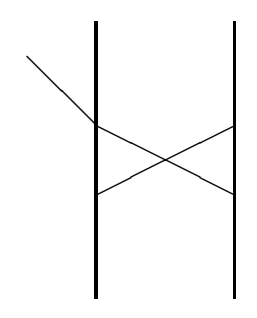

(c)

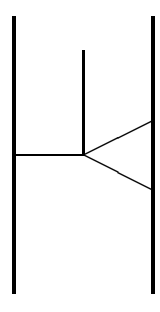

(d)

Fig.2: Selected $\nu=2$ diagrams; the thick (thin) lines represent nucleons (pions).

Let $\mathcal{T}_{n}^{(\nu=2)}(n=1,2 \ldots, 7)$ represent the transition operator coming from the $n$-th type of diagrams. The importance of each operator may be measured in terms of the ratio $\mathcal{R}_{n} \equiv \mathcal{T}_{n}^{(\nu=2)} / \mathcal{T}^{(\nu=1)}$, where $\mathcal{T}^{(\nu=1)}$ is the leading-order two-body transition operator.

Of the seven types of diagrams, some can be interpreted as vertex corrections to the lower order operators, $\mathcal{T}^{(\nu=-1)}$ and $\mathcal{T}^{(\nu=1)}$; Fig.2(a) gives an example. The other types are rather loosely called "two-pion" diagrams; three examples are shown in Figs. 2(b),(c),(d).

We have found [29] that $\mathcal{R}_{n}$ 's corresponding to the vertex-correction-type diagrams are 
small $(0.1 \sim 0.2)$ in conformity with the general expectation of $\chi \mathrm{PT}$. On the other hand, some of the "two-pion" diagrams turn out to give very large contributions. Especially, $\mathcal{R}_{n}$ 's belonging to the types illustrated in Fig.2(b), Fig.2(c) and Fig.2(d) are individually very large. For the first two, $\left|\mathcal{R}_{n}\right|=3 \sim 7$ (depending on the input low-energy parameters), while $\left|\mathcal{R}_{n}\right|=7 \sim 10$ for the pion-pion rescattering diagram, Fig.2(d). This feature is consistent with the expectation that the $p p \rightarrow p p \pi^{0}$ reaction is sensitive to heavymeson exchanges. Thus the phenomenologically important $\sigma$-meson contributions 23] seem to have discernible "representatives" among the NNLO $\chi \mathrm{PT}$ diagrams. The large contributions of the "two-pion" diagrams do not necessarily constitute evidence for the non-convergence of $\chi \mathrm{PT}$ expansion. Since these diagrams appear only at NNLO or higher, the convergence can be tested only by calculating corrections to the NNLO diagrams. On the historical note, I should mention that Gedalin et al. 28 considered some of the NNLO diagrams and pointed out that they could be large. According to our calculation, some important NNLO diagrams are missing in [28] and a loop integral expression in 28] needs to be corrected.

DKMS use the "standard" chiral counting rule of Weinberg [3], whereas Cohen et al. [26] have emphasized that for the $N N \rightarrow N N \pi$ reaction, which involves significant energymomentum transfers, one should modify the chiral counting rule. In this new counting

scheme, the expansion parameter is not any longer $m_{\pi} / m_{\mathrm{N}}$ but $\sqrt{m_{\pi} / m_{\mathrm{N}}}$. It is a future task to perform a calculation similar to that of DKMS using the modified counting rule. Furthermore, to obtain transition amplitudes that can be directly compared with the experimental cross sections, it is imperative to carry out a distorted-wave calculation. We hope to be able to report our investigation along this line in the near future.

In the first part of my talk I have surveyed the recent progress in $\chi \mathrm{PT}$ treatments of low energy-momentum transfer observables in the two-nucleon systems. I have mentioned that we are entering the first stage of $a b$ initio calculations based on $\mathrm{HB} \chi \mathrm{PT}$. It will be extremely nice if we can perform similar ab initio calculations for e.g. $N N \rightarrow N N \pi$, which involve higher energy-momentum transfers. This is a difficult but urgent challenge.

\section{Acknowledgments}

It is my great pleasure to attend this Symposium dedicated to Professor Koichi Yazaki, and I would like to take this opportunity to express my sincere gratitude to Koichi for his warm friendship and his kind guidance in physics for over three decades. The work described here has been carried out in collaboration with Tae-Sun Park, Dong-Pil Min, Mannque Rho, Toru Sato, Veljko Dmitrasinovic and Fred Myhrer. I owe deep thanks to each of these colleagues.

\section{REFERENCES}

1. S. Weinberg, Physica, 6A (1979) 327.

2. J. Gasser and H. Leutwyler, Ann. Phys. 158 (1984) 142; J. Gasser, M. Sainio and S. Švarc, Nucl. Phys. B, 307 (1988) 779.

3. S. Weinberg, Phys. Lett. B, 251 (1990) 288 (1990); Nucl. Phys.B, 363 (1991) 3; Phys. Lett. B, 295 (1992) 114. 
4. E. Jenkins and A.V. Manohar, Phys. Lett. B, 255 (1991) 558.

5. For a review, see e.g., V. Bernard, N. Kaiser and U.-G. Meissner, Int. J. Mod. Phys. E4 (1995) 193.

6. U. van Kolck, thesis, University of Texas (1992); Phys. Rev. Lett. 72 (1994) 1982.

7. K. Kubodera, J. Delorme and M. Rho, Phys. Rev. Lett. 40 (1978) 755.

8. M. Rho, Phys. Rev. Lett. 66 (1991) 1275.

9. T.-S. Park, D.-P. Min and M. Rho, Phys. Rev. Lett. 74 (1995) 4153; Nucl. Phys. A, 596 (1996) 515.

10. T.-S. Park, D.-P. Min and M. Rho, Phys. Reports, 233 (1993) 341; T.-S. Park, I.S. Towner and K. Kubodera, Nucl. Phys. A, 579 (1994) 381.

11. E.K. Warburton, Phys. Rev. Lett. 66 (1991) 1823; E.K. Warburton, I.S. Towner and B.A. Brown, Phys. Rev. C, 49 (1994) 824.

12. T.-S. Park, K. Kubodera, D.-P. Min and M. Rho, Astrophys. J. 507 (1998) 443.

13. J.N. Bahcall and R. May, Astrophys. J. 155 (1969) 501; M. Kamionkowski and J.N. Bahcall, Astrophys. J. 420 (1994) 420; for a recent review, see E.G. Adelberger et al, Rev. Mod. Phys. 70 (1998) 1265.

14. A.N. Ivanov, H. Oberhammer, N.I. Troitskaya and M. Faber, nucl-th/9811012.

15. T.-S. Park, K. Kubodera, D.-P. Min and M. Rho, Phys. Rev. C 58 (1998) R637.

16. T.-S. Park, K. Kubodera, D.-P. Min and M. Rho, Nucl. Phys. A, 646 (1999) 83.

17. G.P. Lepage, nucl-th/9706029.

18. D.B. Kaplan, M.J. Savage and M.B. Wise, Phys. Lett. B, 424 (1998) 390; Nucl. Phys. B, 535 (1998) 329.

19. D.B. Kaplan, M.J. Savage and M.B. Wise, Phys. Rev. C, 59 (1999) 617.

20. M. Savage, K.A. Scaldeferri and M.B. Wise, nucl-th/9811029, and references therein.

21. S.R. Beane, T.D. Cohen and D.R. Phillips, Nucl. Phys. A632 (1998) 445; J.V. Steele and R.J. Furnstahl, Nucl. Phys. A, 637 (1998) 46; J. Gegelia, Phys. Lett. B, 429 (1998) 227; M. Birse, nucl-th/9804028.

22. H. O. Meyer et al. , Phys. Rev. Lett. 65 (1990) 2846; Nucl. Phys. A, 539 (1992) 633; A. Bondar et al. , Phys. Lett. B, 356 (1995) 8.

23. T.-S. H. Lee and D. O. Riska, Phys. Rev. Lett. 70 (1993) 2237.

24. E. Hernández and E. Oset, Phys. Lett. B, 350 (1995) 158; C. Hanhart, J. Haidenbauer, A. Reuber, C. Schütz and J. Speth, Phys. Lett. B, 358 (1995) 21; Acta Phys. Pol. B, 27 (1996) 2893; N. Fettes, U.-G. Meissner and S. Steininger, Nucl. Phys. A, 640 (1998) 199; V. Bernard, N. Kaiser and U.-G. Meissner, nucl-th/9806013 (1998).

25. B.-Y. Park, F. Myhrer, T. Meissner, J.R. Morones and K. Kubodera, Phys. Rev. C, 53 (1996) 1519.

26. T.D. Cohen, J.L. Friar, G.A. Miller and U. van Kolck, Phys. Rev. C, 53 (1996) 2661.

27. T. Sato, T.-S. Lee, F. Myhrer and K. Kubodera, Phys. Rev. C, 56 (1997) 1246.

28. E. Gedalin, A. Moalem and L. Razdolskaya, Ben Gurion Univ. preprint, nuclth/9803029.

29. F. Myhrer, Proc. of the IUCF Workshop on Few-Nucleon Physics with Stored, Cooled Beams (September 1998), page 142;

V. Dmitrasinovic, K. Kubodera, F. Myhrer and T. Sato, nucl-th/9902048. 